\title{
Towards to an Bio-inspired Orchestration of Mobile Learning Activities
}

\author{
Nassim Dennouni, Yvan Peter, Luigi Lancieri \\ NOCE team, LIFL Laboratory, Lille1 University, Lille, France \\ nassim.dennouni@ed.univ-lille1.fr, yvan.peter@univ-lille1.fr, luigi.lancieri@univ-lille1.fr \\ Zohra Slama \\ ISIBA team, EEDIS Laboratory, Djilali Liabes University, Sidi Bel Abbes, Algeria \\ n.dennouni@univ-chlef.dz,slama@univ-sba.dz
}

\begin{abstract}
This paper presents a new approach to a recommendation of learning activities adapted to the spatial and temporal context of each mobile learner. Indeed, the path traveled by the user during a field trip can be guided using the technique of passive collaborative filtering. This recommendation is based on the ACO (Ant Colony Optimization) algorithm, which represents a good model for swarm intelligence. For this reason, the structure of our mobile scenario is described as a graph where POIs (Point Of Interest) are represented by nodes and the arcs indicate the probability of moving between them. This recommendation system allows the orchestration of mobile learning according to the geographical location of learners and the historical of their activities. Our contribution is devised in three parts: (1) the creation of a mobile learning scenario based on POIs, (2) the adaptation of the ACO algorithm for the orchestration of paths taken by learners, and (3) the development of a recommender system that helps learners to better choose their paths during the field trip.
\end{abstract}

Index Terms-Mobile learning, field trip scenario, POI, orchestration of mobile learning activities, recommendation system, passive collaborative filtering, ACO algorithm.

\section{INTRODUCTION}

With the development of mobile technologies and the increasing use of Smartphone, users increasingly integrate these new devices in their learning process. In this context, mobile learning was recommended to motivate students during field trips [1] because it allows constructing a personalized knowledge by interacting with other. However, because of the wealth of learning resources and the growth of comments posted by learners, information overload by POI remains a major concern to guide mobile learning. On the other hand, the geographic orchestration of resources and activities is often done on the basis of POIs locations and it does not consider the global vision of the learning scenario [2]. According to Sharples [3], this kind of learning should be individualized and learner-centered because it is based on an implicit collaborative process that occurs when visitors have problems to solve or knowledge to share. In order to do this, we focus on the recommendation of POI during the field trip to better orchestrate mobile learning activities in the basis of the geographical location of learners and the historic of their visits. Our proposal implements a decentralized orchestration of mobile learning activities using a passive collaborative filtering method based on previous investigations of learners. This approach is inspired of the ACO algorithm that is relatively a new field of artificial intelligence.

In section 2 of this article, we will present different techniques that can be applied to recommend POIs in mobile learning scenario. Next, we define our theoretical context by providing a method for the orchestration of mobile learning activities based on the adaptation of the ACO algorithm. In Section 4 of this article, we present our contribution that contains three elements: 1) the adoption of a scenario based on POIs, 2) the definition of dimensions to integrate into our recommendation system and 3) the description of functioning of our orchestration mechanism. Then, in Section 5, we will discuss the performance results obtained from the simulation of our collaborative filtering algorithm. Finally, we present the details of the design and the implementation of our proposed solution.

\section{LITERATURE REVIEW}

Recommendation systems start with the observation that the main choices of a group of individuals can help to take a decision in a context of uncertainty. This is a form of exploitation of collective intelligence [4]. These systems are widely used to advise contents such as books or music on the basis of the discovery of similarities rules in user's activities [5]. Below, we focus the state of the art on geographical (location or path) recommendation systems since this topic is rather few addressed in the literature. Many systems based on automatic recommendation of POIs can be proposed to facilitate learning during field trips. These systems are based on algorithms that can do the orchestration of mobile activities in the aim to provide a learning path adapted to each learner.

According to Candillier et al., the methods that use a 
collaborative filtering give better results than those using a content filtering [6]. For this reason, we present a review of the main collaborative systems that can recommend POIs for mobile learning scenario.

Valigiani et al. adapt the ACO algorithm to a learning environment organized as a graph of hyperlinks. The structure of this graph can be optimized in order to facilitate the learning process for all users [7]. On the other hand, Wang et al. proposes an alternative approach based on ACO algorithm to help learners to progress in their individualized learning using an adaptive learning path. This type of recommendation identifies the POI that is most likely to be chosen to form an optimal path based on different learning styles [8]. Kurilovas et al. presents a recommendation of a learning path associed to each group of learners. The ACO algorithm is used for the selection and combination of sequences of LOs (Learning Objects) based on the preferences of learners. This recommendation allows to rearrange the alignment of LO using the ACO pheromones in the aim to individualize the learning path [9].

The previous work [7] [8] [9] can be used to orchestrate the learning activities of our mobile scenario thanks to the path marked by pheromone during the visit. However, these studies do not support the temporal context of our learning and do not monitor the sequencing of our educational scenario.

In [10], the authors present a technique that allows an explicit collaborative filtering to better customize the recommendation of POIs. This method is essentially based on all assessments made by the owner of the smartphone as well as the ratings of other users. According to Zheng et al., analysis of the historical of GPS traces relating to ancient visitors, also allows a recommendation of activities on some POIs [11]. On the other hand, Phichaya-anutarat et al. propose a recommendation technique to assist the user in selecting appropriate POIs according to their preferences and interests [12]. This approach uses demographic data such as personal results and users profiles available in the DB (data base) of the system.

These three works [10] [11] [12] are based on explicit collaborative filtering based on the historical obtained after several visits to provide personalized recommendations. However, during the progress of the field trip, learners are implicit in their collaborations. For this reason, collecting relevant information from traces of visited sites and user profiles can be costly and complex. Consequently, this type of recommendation does not seem suited to our learning needs because it neglects the educational dimension of our scenario.

As part of mobile learning scenarios, Ye et al. have developed an algorithm based on naive Bayesian classification. This technique recommends POIs according to user location and social influence [13]. Biancalana et al. describe a recommendation system based on social relationships able to identify preferences of users and their information needs. This system uses a process of filtering information to suggest a personalized recommendation of POIs related to the current location of the user [14].

These two works [13] [14] are within the scope of the recommendations made thanks to the social relationships that may exist between learners. However, the scenario of the field trip starts without historical data because it has no information on the different social interactions. For that, this kind of recommendation seems not well adapted to the spatiotemporal context of the visit.

The work of Sang et al. allows users to schedule activities based on the probability of transition from one POI to another depending on the historical in a Markov chain [15]. On the other hand, Cheng et al. use a personalization of the Markov chain to explore user preference. They social and geographical influence for predict the POI visit from a region relating to a location [16].

These two works [15] [16] can be used to predict the POI to visit during the visit. However, this recommendation is based only on the historical of each learner but it neglects the views of the instructor. The previous orchestrations can be used to recommend resources and activities adapted to spatiotemporal context of mobile learners.

Table 1 summarizes the main characteristics related to the orchestration of mobile learning activities:

Table 1. Characteristics of different type of orchestration

\begin{tabular}{|c|c|c|c|c|}
\hline Works & $\begin{array}{l}7] \\
{[8]} \\
{[9]}\end{array}$ & $\begin{array}{l}10] \\
{[11]} \\
{[12]}\end{array}$ & $\begin{array}{l}{[13]} \\
{[14]}\end{array}$ & $\begin{array}{l}{[15]} \\
{[16]}\end{array}$ \\
\hline $\begin{array}{c}\text { Element } \\
\text { of } \\
\text { Context }\end{array}$ & $\begin{array}{l}\text { Spatio- } \\
\text { temporal } \\
\text { context }\end{array}$ & $\begin{array}{l}\text { GPS tracks } \\
\text { and } \\
\text { interests of } \\
\text { learners }\end{array}$ & $\begin{array}{c}\text { social } \\
\text { context } \\
\text { and location }\end{array}$ & $\begin{array}{l}\text { Historical of } \\
\text { POI visited }\end{array}$ \\
\hline $\begin{array}{c}\text { Interacti } \\
\text { on } \\
\text { between } \\
\text { participa } \\
\text { nts } \\
\end{array}$ & $\begin{array}{l}\text { Clicks } \\
\text { on } \\
\text { hyper- } \\
\text { links }\end{array}$ & $\begin{array}{c}\text { GPS } \\
\text { tracks and } \\
\text { Ratings }\end{array}$ & $\begin{array}{l}\text { Social } \\
\text { relation- } \\
\text { ships }\end{array}$ & $\begin{array}{c}\text { GPS tracks a+ } \\
\text { nd Ratings }\end{array}$ \\
\hline $\begin{array}{l}\text { Real- } \\
\text { time } \\
\text { collabor } \\
\text { ation }\end{array}$ & $\begin{array}{l}\text { Implicit } \\
\text { collabo- } \\
\text { rative } \\
\text { filtering }\end{array}$ & $\begin{array}{l}\text { Explicit } \\
\text { collabo- } \\
\text { rative } \\
\text { filtering }\end{array}$ & $\begin{array}{l}\text { social } \\
\text { collabo- } \\
\text { rative } \\
\text { filtering }\end{array}$ & $\begin{array}{c}\text { Predic-tion of } \\
\text { POIs }\end{array}$ \\
\hline $\begin{array}{c}\text { Method } \\
\text { used }\end{array}$ & $\begin{array}{c}\mathrm{ACO} \\
\text { algorith } \\
\mathrm{m}\end{array}$ & $\begin{array}{c}\text { User- } \\
\text { Centered } \\
\text { Approach }\end{array}$ & $\begin{array}{c}\text { Naive } \\
\text { Bayes } \\
\text { classifier }\end{array}$ & Markov chain \\
\hline $\begin{array}{l}\text { limit of } \\
\text { the } \\
\text { method }\end{array}$ & $\begin{array}{c}\text { The } \\
\text { scenario } \\
\text { depends } \\
\text { on the } \\
\text { hypertex } \\
\text { t } \\
\text { structure }\end{array}$ & $\begin{array}{l}\text { Recomme } \\
\text { ndation } \\
\text { depends } \\
\text { on the } \\
\text { learners' } \\
\text { profiles }\end{array}$ & $\begin{array}{l}\text { Recommen } \\
\text { dation } \\
\text { depends on } \\
\text { social } \\
\text { relations }\end{array}$ & $\begin{array}{l}\text { POI prediction } \\
\text { depends on } \\
\text { the area } \\
\text { visited }\end{array}$ \\
\hline
\end{tabular}

After this state of the art, we found that there are several works on the recommendation of POIs that can be used in the case of field trips. However, our mobile scenario is related to the context of the visit and must be limited to the learning objectives while allowing implicit collaboration between learners. For this reason, we propose later a flexible solution that will support this type of learning activities. 


\section{OUR THEORETICAL CONTEXT}

\section{A. The orchestration in the context of field trip}

Field trips must be designed around specific educational goals. If a trip has not been well planned in advance, it will end in confusion and can cause a loss of time and money.

In order to solve this problem, the visit should be conceived as a cooperative activity involving the participation of students under the supervision of the instructor [17].

Indeed, the scenario of a field trip can be divided into three distinct phases: 1) preparation phase which allows for the planning of field visits, 2) the movement itself as the second phase and 3) the final phase that include follow-up activities such as visit reports, presentations, tests and / or feedbacks.

The first phase is dominated by the work of the teacher. The field visit and the finalization phase are based on the activities of learners. The tour takes place outside the classroom, while the phases of preparation and finalization can occur inside or outside the classroom [18] According to Dillenbourg, orchestration refers to the realtime management of educational graphs at the individual, group, class or community in the aim to incorporate constraints that cannot be expressed in design phase [19]. Specht and Glahn explain that the orchestration also covers personalization and adaptation in a learning environment [20].

In order to solve this problem, we define the POI like the smallest indivisible element of a learning path associated with a location relative to a given reference. Therefore, the POI can be considered as an educational grain associated to a specific location such as an address of a location. For this reason, the POI may contain one or more resources such as text, images, link, animations, videos. and one or more activities such as questions, simulations, etc.

In our context of field trip, the design of mobile scenario for learners relies mainly on the choice of POIs based on educational objectives. However, each learner can choose his own path during the visit. This trajectory reflects the execution of our mobile scenario through the activities associated with each POI.

To solve this problem, we'll use the order of POI to better guide the learners according to the learning objectives as shown in Figure 1.

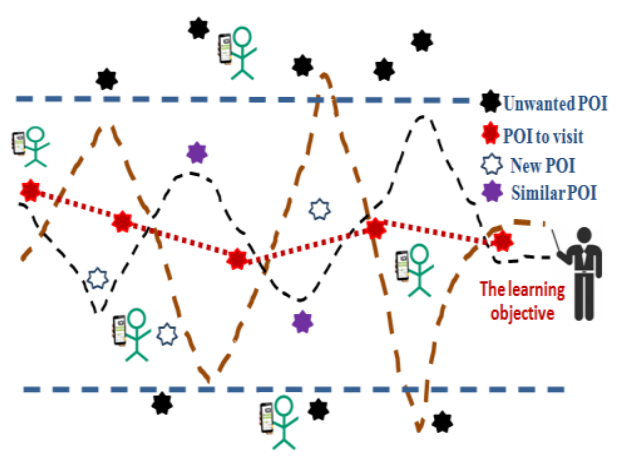

Fig.1. Orchestration of mobile activities by choosing the path associated to the learning objective.

\section{B. Optimization of POI path and ACO algorithm}

The first ACO algorithm [21] aims to solve the traveling salesman problem, where the goal is to find the shortest path for connecting a set of cities. In our work, we found that there is an analogy between the optimization of the traveling salesman problem and the optimization of the path of mobile learners as shown in the table 2:

Table 2. Analogy between optimization of learning path based on POIs and the traveling salesman problem

\begin{tabular}{|l|l|}
\hline \multicolumn{1}{|c|}{$\begin{array}{c}\text { Constraints related to the } \\
\text { traveling salesman problem }\end{array}$} & $\begin{array}{c}\text { Constraints related to the } \\
\text { learning path based on POIs }\end{array}$ \\
\hline Each ant must visit all the cities & Each learner must visit all POIs \\
\hline $\begin{array}{l}\text { Each ant must visit each city } \\
\text { only once. }\end{array}$ & $\begin{array}{l}\text { Each learner must visit each POI } \\
\text { only once }\end{array}$ \\
\hline $\begin{array}{l}\text { If the city is far, it has less } \\
\text { chance of being selected. }\end{array}$ & $\begin{array}{l}\text { If the POI is far, it has less } \\
\text { chance of being selected. }\end{array}$ \\
\hline $\begin{array}{l}\text { If pheromones deposited } \\
\text { between two cities are higher, } \\
\text { this path is more likely to be } \\
\text { chosen }\end{array}$ & $\begin{array}{l}\text { If the link between two POI's is } \\
\text { most frequented by the learners, } \\
\text { this path is more likely to be } \\
\text { chosen }\end{array}$ \\
\hline
\end{tabular}

From each POI, multiple paths are possible but to choose the optimal path (the most frequented by learners), we use the random proportional transition rule as indicated in Figure 2.

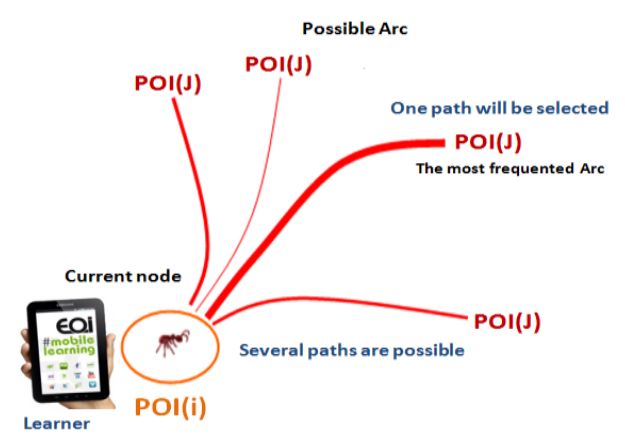

Fig.2. Transition between the current POI and the next POI. 
This rule allowing the displacement between the current POI and the next POI is written mathematically in formula 1:

$$
P_{i j}^{k}(t)=\frac{\left[\tau_{i j}(t)\right]^{\alpha}\left[\mu_{i j}(t)\right]^{\beta}}{\sum_{l \in D_{i}^{K}}\left[\tau_{i l}(t)\right]^{\alpha}\left[\mu_{i l}(t)\right]^{\beta}}
$$

$\mathrm{P}_{\mathrm{ii}}{ }^{\mathrm{k}}(\mathrm{t})$ represents the transition probability of POI (i) to the POI (j) during iteration $t$. This probability depends on the combination of two values: 1) $\tau \mathrm{ij}(\mathrm{t})$ which is the pheromone quantity (intensity) on the $\operatorname{arc}(\mathrm{i}, \mathrm{j})$ at time $\mathrm{t}$ and 2) $\mu \mathrm{ij}(\mathrm{t})$ the value of the visibility which is equal to the inverse of distance between POI (i) and POI (j). In Formula $1, \mathrm{D}_{\mathrm{i}}^{\mathrm{k}}$ represents the list of possible moves for the learner $\mathrm{K}$ when he is on the POI (i). The two main parameters controlling the algorithm are $\alpha$ and $\beta$, which control the relative importance of intensity and a transitional arc visibility. When the path of POIs is made, the learner k lays a quantity of pheromone on each arc of the trajectory. The value of this quantity is calculated in formulas 2 and 3 :

$$
\Delta \tau_{i j}^{k}(t)=\frac{Q}{L^{k}(t)}
$$

If $(i, j) \in \tau^{k}(t)$

$$
\Delta \tau_{i j}^{k}(t)=0
$$

If $(i, j) \notin \tau^{k}(t)$

Where $\tau^{\mathrm{k}}(\mathrm{t})$ is the path taken by the $\mathrm{k}^{\mathrm{th}}$ learner at iteration $t, \mathrm{~L}^{\mathrm{k}}$ is the path length and $\mathrm{Q}$ is a parameter setting.

At the end of each iteration of the algorithm, quantities of pheromones deposited by learners evaporate. This quantity is calculated as follows in formula 4:

$$
\rho \Delta \tau_{i j}^{k}(t)
$$

At the end of each iteration, we can calculate the sum of pheromones that have not evaporated compared with those which have been deposited. This is symbolized by the delta sum in formula 5:

$$
\tau_{i j}^{k}(t+1)=(1-\rho) \tau_{i j}^{k}(t)+\sum_{k=1}^{m} \Delta \tau_{i j}^{k}(t)
$$

Where $m$ (sum parameter) is the number of learners participating in the iteration $t$ and $\rho$ is a parameter setting. The ACO meta-heuristic may have additional behavior that consist to depose additional pheromones "offline" while respecting pheromones "online" filed by learners [22] [23]. For this reason, we make an analogy between the ants colony and the learners class (c) in the aim to propose the following algorithm:

\section{Algorithm Recommendation of the Best Learning Path BEGIN}

1) The instructor chooses an initial path of the visit; (He marks this path of POIs using pheromones matrix)

FOR $\mathrm{N}=1$ to Number of Classes (c) DO

FOR K = 1 to Number of Learners (m) DO

WHILE (Current POI! = Target POI) Do

2) Calculate the probability $P$ of the transitions from the current POI to each POI;

3) Moving to the next POI that correspond to the maximum of the values of $P$;

4) Current POI = Next POI;

\section{END WHILE}

5) Evaluate the cost of the path that include all POIs of the visit;

6) If (this path ameliorates the cost of the learning path) then Deposit a quantity (q1) of pheromone on all arcs visited by the learner (m);

\section{END DO}

7) 7) Determine the best path found by all learners of the class (c);

8) Deposit a quantity (q2) of the pheromone on all arcs belonging to this learning path;

9) Remove a quantity (q3) of pheromone on the arcs that do not belong to this learning path;

\section{END DO}

10) Determine the best path found by all classes; END.

Thereafter, we will use this version of the ACO algorithm to implement our recommendation system of POI.

\section{OUR CONTRIBUTION}

This section describes our recommendation system which uses an adaptation of the ACO algorithm to a passive collaborative filtering based on the previous actions of the learners. It is composed of three parts. The first part describes the design of the learning scenario using POI according to the educational objectives of the field trip. The second part focuses on the definition and combination of different matrices that integrate dimensions as the geographical proximity of the POI, learning scenario or the historical visits as part of our recommendation. The third part explains how our recommendation system works depending on the redundancy of learners paths and according to the realtime calculation (I.e. probabilities of transition from one POI to another).

\section{A. The structure adopted for our scenario}

The behavior of the mobile learners cannot be predicted in detail during the design phase but it can be monitored and adjusted during the visit. To do this, when planning the field trip, we can imagine scenarios that adapt themselves to the particularity of each learning situation. This adaptation should have the ability to integrate any new variants other than those already listed. 
In this context, we divide our mobile scenario into several educational grains according to the educational objectives of the field trip. Then, we associated with each educational grain: 1) an absolute location (with Coordinates) or a relative position (using matrix of Neighborhoods), 2) a description of POI 3) the resources to read and 4) the learning activities to do. Furthermore, it is clear that the order of POI influences the degree of understanding of our mobile learning scenario. According to the learner profile, a path can be chosen based on the specific requirements of the spatiotemporal context and the historical of our visit. However, our scenario can integrate anonymous users (without profile) as part of informal learning. On the other hand, communities of visitors are mono criterion and are implicit in their collaborations. To solve this problem, the path of the learner can be individualized naturally [2] (geographic recommendation) but it may be less relevant for learning. In order to avoid this drawback, the recommendation of an educational path is necessary to ensure respect of the pedagogic constraints of our scenario.

\section{B). Definition of dimensions to be included in our system}

As part of our mobile scenario, we can imagine several types of recommendation according to different criteria sought by the orchestration. According to Ye, user behavior is strongly influenced by the proximity of the POI [24] [25]. Indeed, during a field trip, each student will be interested first by visible POIs in its vicinity. To take into account this reality, we can use, for example, the shortest path to minimize the distance traveled during the visit. But, this criterion is not relevant to the pedagogical orchestration of our scenario. On the other hand, the duration of the visit seems to have more influences than the distance because it includes parameters such as the transition delay between POI, time of locating the POI, time of reading the resources and the completion time of activities, etc. These parameters can give us information about the good or bad conduct of our scenario. For example, a too low duration may indicate disinterest of the learner. Conversely, a long duration can give us an idea about the difficulty of the path taken by learners (not visible POI, inappropriate activity in the location of POI...). These indicators are not sufficient to make a recommendation of a path that respects the educational objectives of the visit.

Therefore, the instructor must provide data that allows to identify all educational paths that can provide a good recommendation for learners during the visit. However, this solution might be too rigid because it refers only to the instructor point of view and ignores the historical of the visit. Indeed, a recommendation that is based on the path approved by the majority of learners seems interesting because it respects the point of view of visitors. For this, we defined the following dimensions:

1) The Neighborhood dimension is represented by $V$, a square symmetric matrix of dimension $\mathrm{N}$ ( $\mathrm{N}$ being the number of POI). The values of this matrix represent the measurements of distances between the POIs located in the rows and POIs which are in columns according to an Euclidean metric.

2) The scenario dimension is represented by $S$, an asymmetric square matrix of dimension $\mathrm{N}$ ( $\mathrm{N}$ being the number of POI). In this matrix, the rows represent the location of learners and the columns indicate possible transitions to next POIs. These transitions are executed according to the graph of mobile learning activities designed by the instructor of field trip.

3) The weight dimension is represented by $P$, an asymmetric square matrix of dimension $\mathrm{N}$ ( $\mathrm{N}$ being the number of POI). This matrix shows the number of frequentation of an arc $(i, j)$ which is incremented each time that a learner passes from POI (i) to POI (j)

4) The pheromone is represented by $\mathrm{PH}$, an asymmetric square matrix of dimension $\mathrm{N}$ ( $\mathrm{N}$ being the number of POI). This matrix is updated each time a learner takes a path identical to one of educational paths indicated in the scenario matrix.

5) The probability dimension PR is represented by an asymmetric square matrix of dimension $\mathrm{N}$ ( $\mathrm{N}$ being the number of POI). This matrix is updated based on the values of the previous matrices to calculate in real time the probability of the transition from a POI to another as it's indicated in Figure 3:

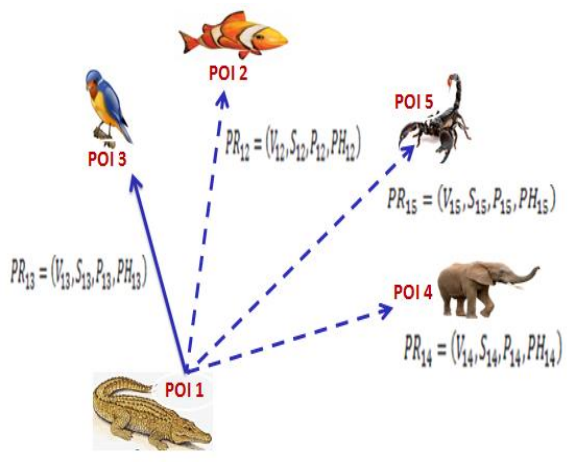

Fig.3. For example, in a context of zoo visit, the visitor can choose his path based on transition probabilities between POIs.

\section{C). Description the functioning of our system}

Field trip requires a user centered scenario based on active learning in order to achieve formal objectives of the visit. In this context, the orchestration done by the instructor cannot manage in real-time the mobile learning activities of each visitor.

Indeed, each educational objective corresponds to a specific set of POI to visit. Therefore, our recommendation system must allow the orchestration of mobile learning activities depending on the redundancy of learning path. This orchestration can recommend to each learner a personalized path.

This order of POIs reflects a scenario based on a sequence of resources to read and activities to do during the visit. In our method, we propose the use of matrices $\mathrm{V}$, $\mathrm{P}, \mathrm{S}$ and $\mathrm{PH}$ for calculating the PR matrix that contains the relative probabilities to the various rules of movement between the POIs. These rules are based on the ACO 
algorithm and they are written mathematically as it's indicated in formula 6 :

$$
P R_{i j}^{k}(t)=\frac{V_{i j}(t)^{\gamma} * P_{i j}(t)^{\alpha} * S_{i j}(t)^{\beta} * P H_{i j}(t)^{\vartheta}}{\sum_{l \in D} V_{i l}(t)^{\gamma} * P_{i l}(t)^{\alpha} * S_{i l}(t)^{\beta} * P H_{i l}(t)^{\vartheta}}
$$

Where $\alpha, \beta, \gamma, \theta$ are the setting parameters for integrating of: 1) the location of POIs, 2) the historical of the visit, 3) the pedagogical scenario and 4) the pheromones that are used in passive collaborative filtering. On the other hand, we suppose that the student is free to follow or not the proposed recommendation by our mechanism. This recommendation is based on a percentage estimated by the instructor that determines the probability of approval of choice of POI recommended. If our learner does not approve the choice of the recommended POI, our system must be able to integrate this action in the historical of our system.

In this paper, we'll focus on a solution selected by the instructor and approved by the learners. So, we will choose among the possible learning paths, the trajectory which seems to be the most frequented by users. For this purpose, we will ignore the constraint of neighborhood assuming that $\gamma=0$ in the calculation of transition rules between the POI because this parameter should not interfere in the context of our recommendation. Our algorithm makes it possible to define a behavior model that can display all POI likely to interest the visitor as part of our system. Then, the learner selects manually the POI to visit depending on its historical activities during the field trip based on the formula 7 :

$$
P R_{i j}^{k}(t)=\frac{P_{i j}(t)^{\alpha} * S_{i j}(t)^{\beta} * P H_{i j}(t)^{\vartheta}}{\sum_{l \in D} P_{i l}(t)^{\alpha} * S_{i l}(t)^{\beta} * P H_{i l}(t)^{\vartheta}}
$$

Where $\alpha, \beta, \theta$ are the setting parameters that define three main classes of recommendation:

a) RecSM (Recommendation of the Solution of Majority)

This type of recommendation is based on the weight of arcs of transition. This value of weight is incremented each time a user moves from one POI to another. In the context, the values of the matrix PR are calculated according to the following parameters: $\alpha=1$ and $\beta=\theta=0$.

b) PRec (Pedagogical Recommendation)

This recommendation is essentially based on the learning scenario. Indeed, from a given POI, our system recommends one or more POI according to the graph of learning activities designed by the instructor of the visit. In this case, the values of matrix PR correspond to the following parameters: $\alpha=0, \beta=1$ and $\theta=0$

c) $\quad \operatorname{Rec}$ (Collaborative Recommendation)

This variant of recommendation uses the values of the $\mathrm{PH}$ matrix obtained from the implicit collaboration of learners. Indeed, this value enhances quality of the learning path proposed by learners. For this technique, we use the following parameters: $\alpha=0, \beta=0$ and $\theta=1$

d) HRec (Hybrid Recommendation)

The instructor of field trip can combine between the values of these three parameters $(\alpha, \beta, \theta)$ to propose new types of recommendation. For example, the combination $\alpha=2, \beta=1$ and $\theta=0$ gives more importance to the history of learners $(\alpha=2)$ relative to the pedagogical scenario $(\beta=1)$ and completely ignores the implicit cooperation $(\theta=0)$. It is clear that this type of recommendation may offer several paths to learners according to the considerations made by the instructor. To evaluate the relevance of the educational paths traveled by learners in the context of a hybrid recommendation or others types of recommendation, we propose the following method:

After completing the term review of all the POIs within our mobile scenario, our system calculates for each learner " $i$ " the precision " $\mathrm{P}_{\mathrm{i}}$ " from the sum of $\mathrm{DH}$ (Hamming distances) between the PFL ( Path Followed by Learner ) and all PDI (Paths Desired by the Instructor) using the formula 8 :

$$
P_{i}(P F L, P D I)=\sum_{k=1}^{N} D H\left(P F L, P D I_{k}\right)
$$

Where $\mathrm{N}$ is the number of educational paths desired by our scenario. This measure assesses the educational relevance of path followed by the learner " $i$ " relatively to the paths indicated by the instructor. It is also a good way to compare the paths traveled by different learners between them. This value can be used as an indicator of performance because it also allows to compare different types of recommendation.

\section{SimUlation RESUlts AND DisCUSSIONS}

In our work we conducted a series of tests to examine the effectiveness of each recommendation proposed by our system based on metrics such as the Levenshtein distance or Hamming distance.

These measures are used to assess the similarity between paths recommended by our algorithm and paths desired by our scenario.

In this context, we addressed the following questions:

A. How the number of learners can influence the behavior of our system?

B. How to compare different approaches of recommendation between them?

C. What is the approach which allows for the minimum dispersion between paths of learners?

D. What is the best approach to use in the recommendation part of our learning process?

E. What is the best rate of approval of the recommendation to associate to our system?

In order to answer to these questions, we developed a 
simulator for the recommendation of learning path that integrates parameters $\alpha, \beta, \theta$ in the calculation of the probabilities of transitions between the POIs.

This simulator allows to recommend a path for each new learner and it also allows varying the number of learners and the approval rate linked to recommended choices. Thereafter, we will assess the suitability of different types of recommendations to our context of field trip.

For this reason, we decided to study the variation of the Hamming distance (between path recommended by our system and the path imposed by the instructor) depending on the number of learners in order to compare the different variants of recommendation described previously.

\section{A). The impact of the RecSM}

At first, we looked at the gap called E_RecSM between the path recommended by the majority of learners and the path desired by the instructor as shown in Figure4:

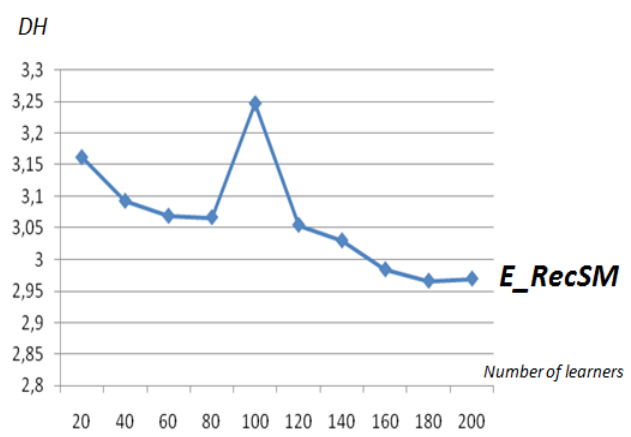

Fig.4. Variation of the gap between the learner path and the path recommended by RecSM

We noticed that the average of gaps called M_E_RecSM = 3.06407 varies considerably depending on the number of learners. So we can deduce that this type of recommendation little adapts to our needs because it is heavily influenced by the natural behavior of learners.

\section{B). The impact of the PRec}

In the second time, we calculated the gap called E_ PRec between the pedagogical paths recommended by the scenario and the path desired by the instructor as it is shown in Figure 5:

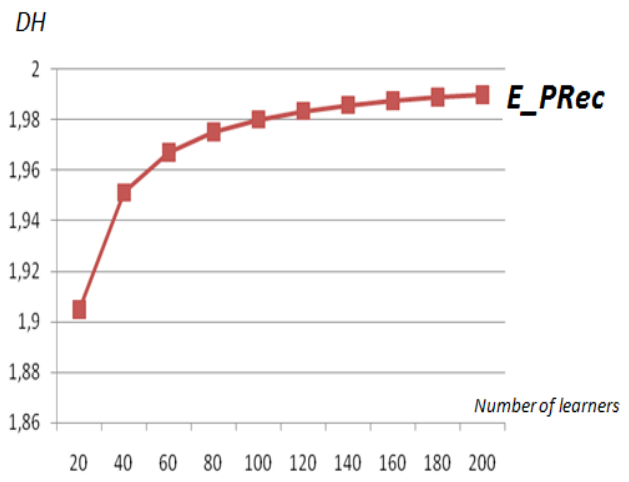

Fig.5.Variation of the gap between the learner path and the path recommended by PRec
We found that the average of gaps M_E_Prec = 1.97145 is well below M_E_RecSM. So this type of recommendation is better suited to our learning process because it respects the objectives of the teacher. However, this type of recommendation is too oriented by the choices imposed by the teacher. Therefore, it fits little active pedagogy used during the course of our field trip scenario.

\section{C). The impact of the CRec}

Finally, we have shown that this type of recommendation varies depending on the collaboration between learners. It is possible to reduce or increase the gap called E_CRec between the paths found by learners and desired paths by the instructor as shown in Figure 6:

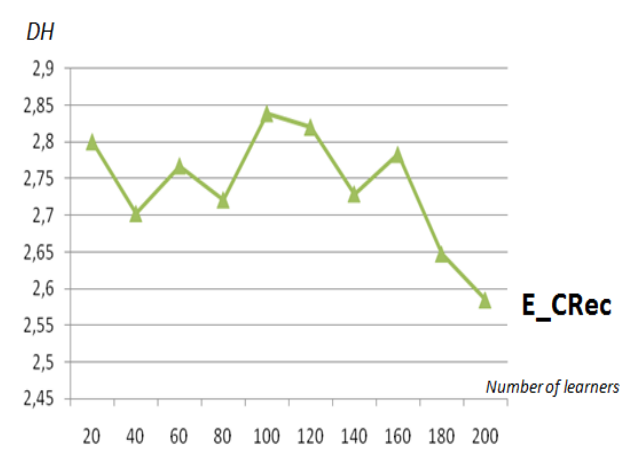

Fig.6.Variation of the gap between the learner path and the path recommended by CRec.

It is clear that the average gap M_E_CRec $=2.7393$ is located between M_E_RecSM = 3.06407 and M_ $\mathrm{E} \_\mathrm{PRec}=1.97145$. So this type of recommendation is well suited to the specificities of our scenario because it allows to set up active learning based on implicit collaboration of learners.

On the other hand, this type of recommendation can provide a solution that is better than the solution proposed by the majority of learners and close to the solution required by the teacher as it is shown in Figure 7

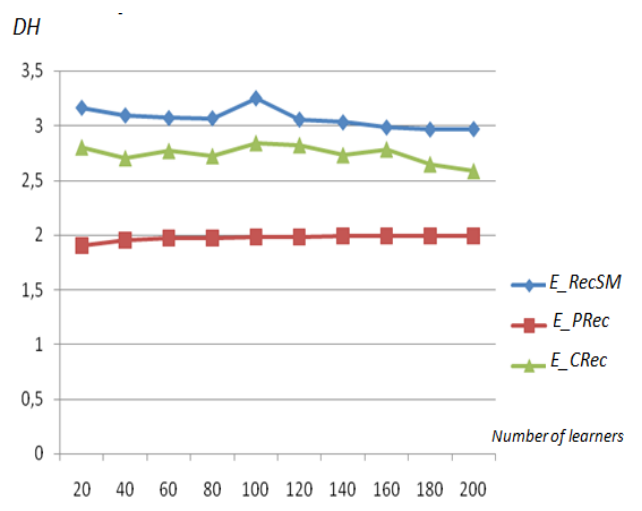

Fig.7. Comparison between RecSM, PRec and CRec

To improve the precision of this recommendation, we proposed two styles of implicit cooperation:

a) The first style allows learners who have taken a path that minimizes the gap E_CRec_DH $\mathrm{DH}_{\text {min }}$ 
between the recommended path and path desired by the teacher to update the PH matrix.

b) The second collaborative style allows only to students who have walked a path identical to one of the paths indicated by the teacher $\left(\mathrm{E} \_\right.$CRec_DH ${ }_{0}$ to change the values of the $\mathrm{PH}$ matrix.

Figure 8 compares the precisions relative to the three variants of recommendation namely E_CRec, E_CRec_DH ${ }_{\min }$ and E_CRec_DH 0

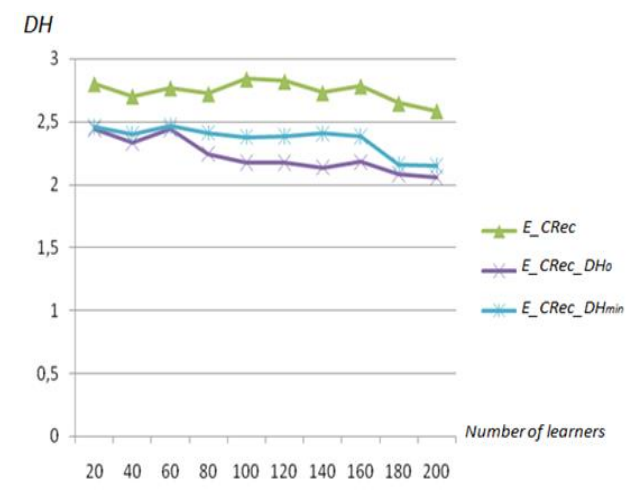

Fig.8. Comparison between CRec, CRec_DH0 and CRec_Dhmin

It is clear that the CRec_DH $\mathrm{DH}_{0}$ has better precision than the $\mathrm{CRec}$ or $\mathrm{CRec} \mathrm{DH}_{\min }$ because it relies on the collaboration of learners who have followed the path suggested by the teacher and ignores any other style of collaboration.

Subsequently, Table 3 compares the standard deviations for the three variants of cooperation mentioned above:

Table 3. Comparison of the dispersion of learners between the three types of collaborative recommendation.

\begin{tabular}{|c|c|c|c|}
\hline NB & o_CRec & o_CRec_DH & $\sigma_{-}$CRec_DH ${ }_{\text {min }}$ \\
\hline 20 & 0,500340020 & 0,498321698 & 0,341325208 \\
\hline 40 & 0,312093479 & 0,249581579 & 0,332280710 \\
\hline 60 & 0,206901151 & 0,390761967 & 0,307878765 \\
\hline 80 & 0,431420200 & 0,283395414 & 0,398340305 \\
\hline 100 & 0,348649839 & 0,133490089 & 0,295706624 \\
\hline 120 & 0,201414904 & 0,129494167 & 0,283090525 \\
\hline 140 & 0,312429035 & 0,133597980 & 0,310778959 \\
\hline 160 & 0,228607433 & 0,221970890 & 0,234369993 \\
\hline 180 & 0,334960367 & 0,051880123 & 0,117886738 \\
\hline 200 & 0,418010465 & 0,029367753 & 0,143335781 \\
\hline Moy_o & 0,329482689 & 0,212186166 & 0,276499361 \\
\hline
\end{tabular}

We note that the $\mathrm{CRec} \_\mathrm{DH}_{0}$ has a better average of standard deviations than other types of recommendations. Therefore, it guarantees less geographic dispersion in groups of learners.

This is explained by the fact that collaboration involves only learners who follow one of the paths indicated by the instructor.

\section{IMPLEMENTATION OF OUR RECOMMENDATION SYSTEM}

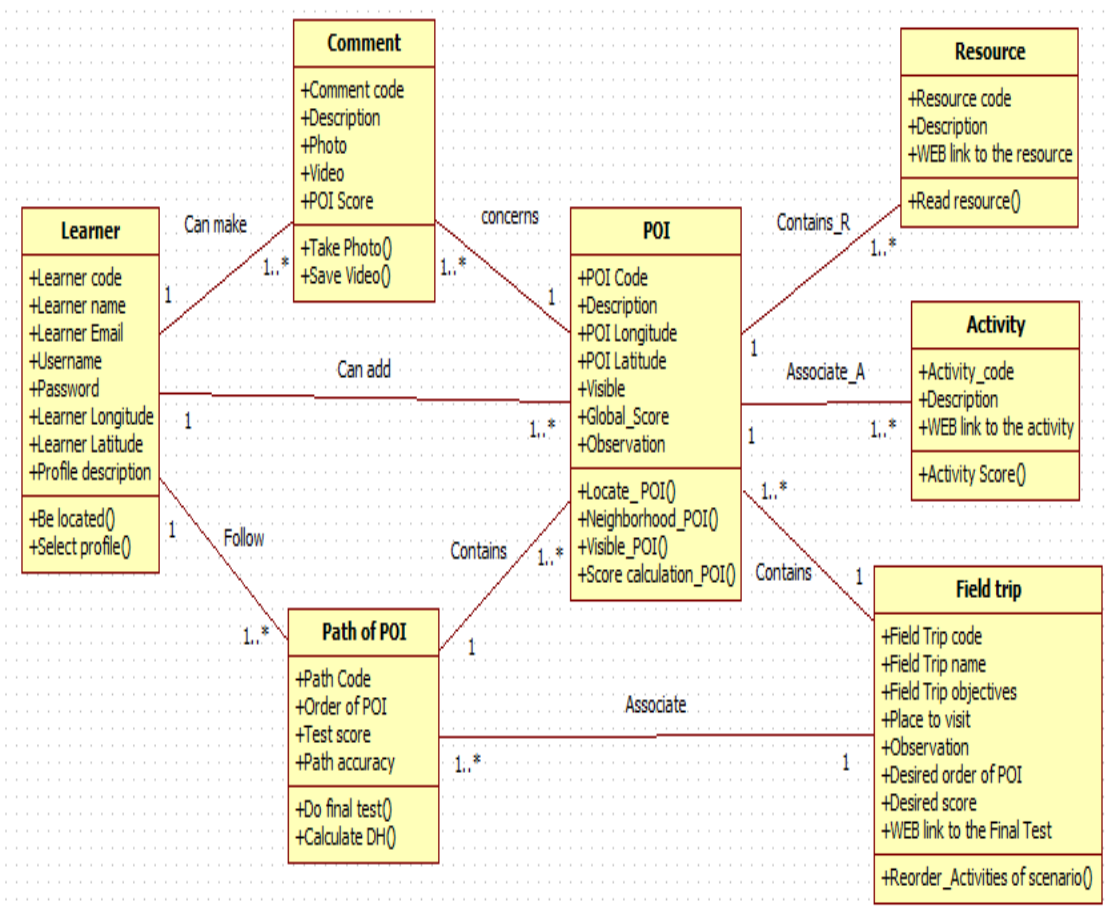

Fig.9.UML class diagram relating to the design of our scenario of field trip

The choice of the software framework depends of our orchestration model of mobile learning activities that are closely linked to the geographical location of the learner and the historical of the visit. 
In general, the main platforms of learning do not allow teachers to anchor the activities or resources in the right places during the field trip. In order to solve this problem, we implemented a new mobile learning environment to provide an automatic orchestration of resources and activities based on the implicit collaboration of learners.

In this context, we have developed a platform that integrates several types of collaboration such as: adding new POIs, the interaction of learners by using comments, ranking the popularity of $\mathrm{POI}$, enrichment of resources for existing POIs, taking pictures for the various POI, registration of explanatory video, etc.

Moreover, this prototype is able to save the traces on different paths traveled by learners and the scores obtained during learning activities.

Our proposed orchestration is collaborative and highly pedagogic because it uses the recommendation called CRec_DH0 which allows learners to fully enjoy the benefits of active learning.

Figure 9 shows the UML class diagram of our prototype:

Our prototype is a web application based on HTML5, CSS, JavaScript, jQuery and the Google Maps API V3. Unfortunately the functionality of Smartphone such as the camera, vibrator, GPS ... are native functions and highly dependent on their operating system. For this reason, we used the PhoneGap framework for creating mobile applications for different platforms (Android, iOS, Windows Phone ...) For example, to take a picture, we use the JavaScript function "camera.getPicture()" in an HTML page and PhoneGap framework will take care to use the correct command line in native language according to the operating system of the Smartphone.

At the WEB server, all data is stored in a database like MySQL and the interaction of the user with our system is achieved through dynamic Web pages using PHP and Java scripts. The algorithm for the recommendation of POI was coded in Java 1.7 and it can be invoked from the Apache server via a web service. Figure 10 depicts the software architecture of our prototype:

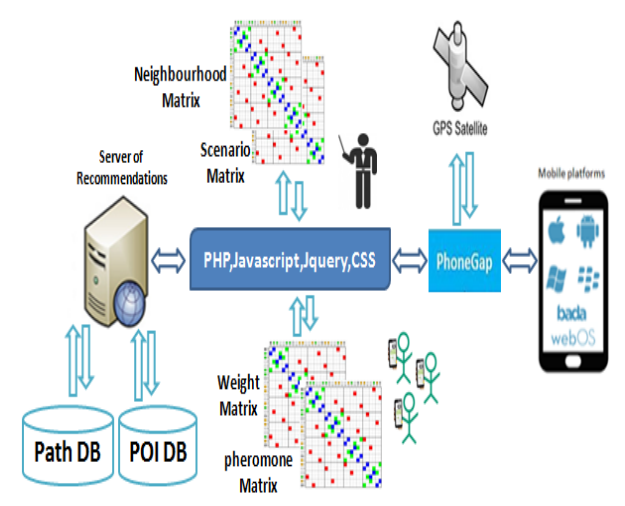

Fig.10.Description of the software architecture relating to our prototype

In order to validate our approach to develop field trip applications, we have implemented a project in aim to discover campus by new students. This prototype allows to help learners to find POIs that are susceptible to be associated to their academic lives. Figure 11 describes the interface that allows the learner to choose between POIs recommended by our system and POIs visible in the map.

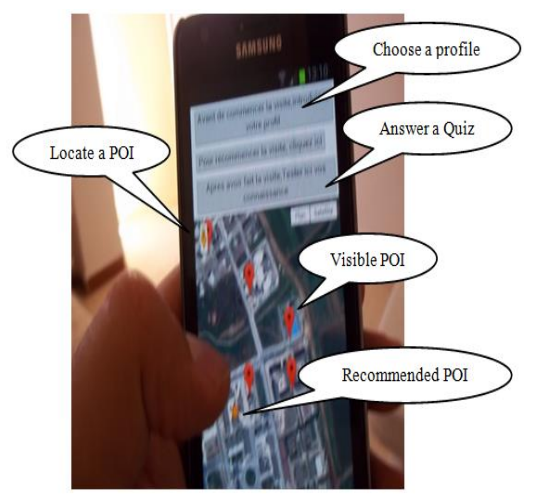

Fig.11. POIs proposed in the personalized context of learner

\section{CONCLUSION}

Our objective was to define a recommendation system that allows orchestration of mobile learning depending on the geographical location of the learner and his historical of activities.

In this article, we designed an adaptive scenario based on POI that allows the instructor to guide the students during the field trip. Then, we established a mechanism of orchestration that can provide to learners with the resources to read and the activities to do depending on the order of traversal of POI. This sequence of resources and activities is needed to better understand the educational objectives of our situated learning. Therefore, we integrated several dimensions such as weight, Scenario and Collaboration to offer a POI recommendation according to the spatio-temporal context of the learner

In this context, we tested and compared several variants of recommendation to choose the best way to adapt our orchestration to the specificities of the active learning. Indeed, our recommendation system can be configured by the instructor to make the good collaborative filtering of POIs as part of our scenario of field trip. This system allows a mobile learning that adapts in real time with the location and historical of the learner.

In the end of our article, we presented an implementation of our UML class diagram to validate our approach destined to the design and the implementation of our scenario. However, our system has some limitations for the integration of all the constraints related to the scenario of the field trip and for identifying different possible learning paths.

Furthermore, we noted that our recommendation mechanism strongly depends on the level of repeal of learners but it neglects the score obtained by the learners during learning activities.

Our system is now in test because it is tested in the context of discovery of campus by new students. Testers of our application emphasize the simplicity of its use and usefulness of the recommendations provided in real time. Future works planned as part of this experiment include: 
1) Identification of learner profiles

2) Adding new attributes to the learning orchestration such as the results of activities, the score of assessments, etc.

3) Integration of an additional context for the recommendation, for example, weather conditions, the state of the user (by foot, car, etc.)

4) The empirical evaluation of user experience with our mobile application.

\section{REFERENCES}

[1] Hung, P.H., Hwang, G.J., Lin, Y.F., Wu, T.H. and Su, I.H.: Seamless Connection between Learning and Assessment-Applying Progressive Learning Tasks in Mobile Ecology Inquiry, Educational Technology \& Society. ISSN 1436-4522 194-205.

[2] Dennouni, N., Peter, Y., Lancieri, L. and Slama, Z.: To a Geographical Orchestration of Mobile Learning Activities. iJIM International Journal of Interactive Mobile Technologies. ISSN: 1865-7923, Volume 8, Numéro 2, (2014) 35-41

[3] Sharples, M.: The design of personal mobile technologies for lifelong learning, Computers \& Education Volume 34 (2000) 177-193

[4] Lancieri, L., : Collective intelligence in a computer mediated environment, in Handbook of Research on Democratic Strategies and Citizen-Centered EGovernment Services, Ejub Kajan editor, IGI global (2014)

[5] Lancieri, L., Manguin, M. and Mangon; S.,: Evaluation of a recommendation system for musical contents, IEEE International Conference on Multimedia \& Expo, Hannover (ICME 2008)

[6] Candillier, L., Jack, K., Fessant, F. and Meyer, F.: Stateof-the-art recommender systems, Collaborative and Social Information Retrieval and Access, (2009).

[7] Valigiani, G., Jamont, Y. and Bourgeois Republique, C.: Experimenting with a Real-Size Man-Hill to Optimize Pedagogical Paths, ACM Symposium on Applied Computing. (2005).

[8] Wang, T., Wang, K. and Huang, Y.: Using a style-based ant colony system for adaptive learning, Available online at www.sciencedirect.com, Expert Systems with Applications pp 2449-2464 (2008).

[9] Kurilovas, E., Zilinskiene, I., and Dagiene, V.: Recommending suitable learning scenarios according to learners preferences: An improved swarm based approach, Elsevier Computers in Human Behavior (2013)

[10] De Spindler, R., Spindler, D., Norrie, M.C., Grossniklaus, M. and Signer, B.: Spatio-Temporal Proximity as a Basis for Collaborative Filtering in Mobile Environments (2006).

[11] Zheng, W.V., Cao, B., Zheng, Y., Xie, X. and Yang, Q.: Towards mobile intelligence: Learning from GPS history data for collaborative recommendation. (2012) 17-37

[12] Phichaya-anutarat, P. and Mungsing, S. : Hybrid recommendation technique for automated personalized POI selection, International journal of information technology (2014)

[13] Picot-Clémente, R. and Bothorel, C. "Un système de recommandation de lieux basé sur la mesure de Katz dans les réseaux sociaux géographiques" MARAMI2013 4ième conférence sur les modèles et l'analyse des réseaux:
[14] Approches mathématiques et informatiques papier publié dans hal-00960139, version 1(2014).

[15] Biancalana, C., Gasparetti, F., Micarelli, A. and Sansonetti, G., :An Approach to Social Recommendation for Context-Aware MobileServices, ACM Transactions on Intelligent Systems and Technology, Vol. 4, No. 1, Article 10, Publication date: January 2013, Copyright 2011 ACM 978-1-4503-0757-4/11/07 pp 325-334(2011).

[16] Sang, J., Mei, T., Tao Sun, J., Xu, C. and Li, S.: Probabilistic Sequential POIs Recommendation via Check-In Data, ACM SIGSPATIAL GIS '12, Nov. 6-9, 2012. Redondo Beach, CA, USA Copyright 2012 ACM ISBN 978-1-4503-1691-0/12/11(2012).

[17] Cheng, C., Yang, H., Lyu, M. R. and King, I.: Where You Like to Go Next: Successive Point-of-Interest Recommendation, Proceedings of the 23 International Joint Conference on Artificial Intelligence, August 3-92013, Beijing, china pp 2605-2611(2013).

[18] Krepel, W.J. and DuVall, C.R.: Field trips, "A guide for planning and conducting educational experiences", National Education Association, Washington, DC, (1981).

[19] Giemza, A., Bollen, L., Seydel, P., Overhagen, A. and Ulrich Hoppe, H.: LEMONADE: "A Flexible Authoring Tool for Integrated Mobile Learning Scenarios" The 6th IEEE International Conference on Wireless, Mobile, and Ubiquitous Technologies in Education, IEEE Computer Society. pp 73-80 (2010).

[20] Dillenbourg, P. :Modéliser l'orchestration, Conférence sur Éducation et sciences de l'apprendre Session 4, Ecole Normale Supérieure de Lyon, automne, (2012).

[21] Glahn, C. and Specht, M. "Embedding moodle intoubiquitous computing environments". In Publications and Preprints. LMedia, (2010).

[22] Dorigo, M., Maniezzo, V. and Colorni, A.: Ant system: optimization by a colony of cooperating agents. IEEE Transactions on Systems, Man, and Cybernetics-Part B, volume 26, numéro, pp. 29-41(1996).

[23] Stutzle, T., Lopez-Ibanez, M., Pellegrini, P., Maur, M., Montes de Oca, M., Birattari, M. and Dorigo, M.: Parameter Adaptation in Ant Colony Optimization, Technical Report No.TR/IRIDIA/2010-002 ISSN 17813794 (2010).

[24] Roy, S. and Chaudhuri, S.: Bio-inspired Ant Algorithms: A review, I.J.Modern Education and Computer Science, ISSN: 2075-0161, volume 4, pp 25-35 (2013)

[25] Ye, M., Yin, P., Lee, W.C. and Lee, D.L.: Exploiting geographical influence for collaborative point-of-interest recommendation, New York, USA, ACM, pp. 325-334 (2011).

[26] Zafar, A. and Hasan, S.: Towards Contextual Mobile Learning, I.J. Modern Education and Computer Science, ISSN: 2075-0161, volume 12, pp 20-25 (2014).

\section{Authors' Profiles}

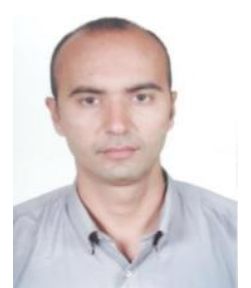

Nassim DENNOUNI is a PhD student in computer science and member in the NOCE team of the LIFL laboratory at Lille 1 university. He is also member in the ISIBA team of the EEDIS laboratory at the Djilali Liabes university (nassim.dennouni@ed.univ-lille1.fr) 


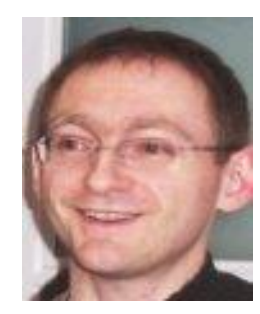

Yvan PETER is an associate Professor in computer science and member in the NOCE team of the LIFL laboratory at Lille 1 university (Yvan.Peter@univ-lille1.fr)

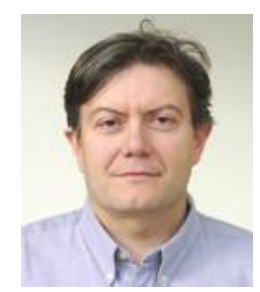

Luigi LANCIERI is a Professor of Computer Sciences and the Head of the NOCE team of the LIFL laboratory at Lille1 university (Luigi.lancieri @univlille1.fr)
Zohra SLAMA is an associate professor in computer science and member in the ISIBA team of the EEDIS laboratory at the Djilali Liabes university of Sidi Bel Abbes. (slama@univsba.dz)

How to cite this paper: Nassim Dennouni, Yvan Peter, Luigi Lancieri, Zohra Slama,"Towards to an Bio-inspired Orchestration of Mobile Learning Activities", IJMECS, vol.7, no.4, pp.1-11, 2015.DOI: 10.5815/ijmecs.2015.04.01 